\section{Tendência secular da idade da menarca avaliada em relação ao índice de massa corporal}

\author{
Secular trends in age at menarche in relation to body mass index
}

Silvia Diez Castilho', Caroline Damasceno Pinheiro', Caroline Agnelli

Bento', Antônio de Azevedo Barros-Filho², Monize Cocetti ${ }^{3}$

\section{RESUMO}

Objetivo: Avaliar a tendência secular da menarca de acordo com o índice de massa corporal (IMC). Sujeitos e métodos: Seiscentos e oitenta e cinco meninas (7-18 anos) avaliadas em 2001 foram comparadas a 750 avaliadas em 2010. Elas foram agrupadas pelo Z-escore do IMC em: (magreza + eutrofia) e (sobrepeso + obesidade). A menarca foi relatada pelo status quo e a idade, estimada pelo logito. Foram utilizados os testes Qui-quadrado, Mann-Whitney e a Regressão logística, com significância de 5\%. Resultados: A menarca adiantou 3,24 meses entre 2001 e 2010. Houve aumento da obesidade e diminuição de eutróficas. 0 evento antecipou 1,44 mês no grupo magreza + eutrofia e 5,76 meses no sobrepeso + obesidade. Não houve interação entre os efeitos determinados pelo período avaliado e diagnóstico nutricional. Conclusões: Embora tanto o período quanto o IMC tenham influenciado a menarca, não se pode atribuir essa antecipação só à mudança do perfil nutricional da amostra. Outros fatores não testados podem estar contribuindo também para isso. Arq Bras Endocrinol Metab. 2012;56(3):195-200

\section{Descritores}

Menarca; índice de massa corporal; obesidade; tendências

\begin{abstract}
Objective: To evaluate the secular trend of menarche according to body mass index (BMI). Subjects and methods: Six hundred and eighty five girls (7-18 years) assessed in 2001 were compared with 750 evaluated in 2010. They were grouped by BMI Z-score: (thin + normal) and (overweight + obese). Menarche was reported by status quo and age at menarche estimated by a logit model. We used the Qui-square test, Mann-Whitney test, and Logistic Regression, at a 5\% significance level. Results: Menarche advanced 3.24 months. There was an increase in obesity, and a decrease of the prevalence of normal girls. Menarche was anticipated by 1.44 month in the thin + normal group and by 5.76 months in the overweight + obese group. There was no interaction between the effects determined by the evaluated period and nutritional diagnosis. Conclusions: Although both the period and BMI influence the menarche, one cannot attribute this advance only to changes in the nutritional profile of the sample. Other factors that were not tested may also contribute to this finding. Arq Bras Endocrinol Metab. 2012;56(3):195-200
\end{abstract}

Keywords

Menarche; body mass index; obesity; trends
1 Faculdade de Medicina, Pontifícia Universidade Católica de Campinas (PUC-Campinas), Campinas, SP, Brasil 2 Departamento de Pediatria, Faculdade de Ciências Médicas, Universidade Estadual de Campinas (Unicamp), Campinas, SP, Brasil ${ }^{3}$ Universidade Federal de São Paulo (Unifesp), São Paulo, SP, Brasil

Correspondência para: Silvia Diez Castilho Av. Princesa D'Oeste, 1144, ap. 171 13100-040 - Campinas, SP, Brasil sdiezcast@puc-campinas.edu.br sdiezcast@gmail.com sdiezcast@hotmail.com

Recebido em 28/Out/2011 Aceito em 13/Fev/2012

\section{INTRODUÇÃO}

$\mathrm{A}$ menarca corresponde a um evento tardio da puberdade e é importante indicador da maturação sexual. A tendência à antecipação da idade em que ela ocorre, observada por décadas nos países desenvolvidos, sempre foi relacionada à melhora das condições de vida e saúde da população, particularmente do acesso aos alimentos (1-3). 
O consumo de alimentos industrializados, ricos em carboidratos e gorduras saturadas, e a mecanização, com consequente diminuição da atividade física, têm levado à deterioração nutricional com aumento da prevalência de excesso de peso. Esse problema a princípio restrito à América do Norte e ao Oeste da Europa se tornou pandêmico e, atualmente, atinge também países subdesenvolvidos e em desenvolvimento, principalmente nos centros urbanos (4-6).

Está bem estabelecido que por questões hormonais o excesso de peso adianta a puberdade nas meninas, fazendo com que elas consequentemente menstruem mais cedo $(7,8)$. Assim, estudos recentes têm sugerido que a idade da menarca pode estar se estabilizando, sendo a antecipação percebida decorrente do aumento da prevalência de sobrepeso e obesidade e não mais relacionada à melhora nutricional (2,9-11).

O impacto do excesso de peso na puberdade se reveste de importância na medida em que a obesidade é fator de risco para doenças cardiovasculares, metabólicas e neoplásicas na idade adulta (12). Por antecipar a menarca, favorece também problemas psicossociais, tais como o início precoce de ingestão de bebida alcoólica e da atividade sexual, aumentando a probabilidade de doenças sexualmente transmissíveis e gravidez na adolescência $(12,13)$.

Diante dessa realidade e da escassez de estudos sobre a tendência da menarca em diferentes segmentos da população no Brasil, este estudo pretendeu avaliar a idade da menarca de acordo com o estado nutricional em meninas que frequentam escolas particulares de Campinas-SP. Esses dados foram comparados aos colhidos há uma década para verificar se continua havendo uma tendência real ou se a antecipação se deve ao aumento da prevalência do sobrepeso e da obesidade.

\section{SUJEITOS E MÉTODOS}

A amostra analisada (1.435 meninas) faz parte de dois estudos transversais cujos dados foram colhidos entre março de 2001 e março de 2002 (685 meninas) - referida como amostra colhida em 2001 - e de fevereiro a novembro de 2010 ( 750 meninas). As meninas tinham entre 7 e 18 anos e frequentavam escolas particulares de Campinas-SP. Foram excluídas as que referiram gravidez, doenças não corrigidas que interferissem no crescimento ou ganho de peso, e as que apresentavam condição que prejudicasse a obtenção das medidas. O tamanho da amostra foi calculado de modo que as adolescentes se distribuíssem por estágio de maturação das mamas segundo os critérios de Tanner. A partir de dados de estudos realizados em adolescentes brasileiros $(14,15)$, calculou-se a idade média para cada estágio maturacional, e, para essa idade, a variabilidade do índice de massa corporal (IMC), utilizando-se a fórmula do tamanho amostral para média de uma variável quantitativa (no caso o IMC) de um estudo descritivo $n=[(Z \times S) / d]^{2}$. Assim, foram consideradas a maior estimativa de desvio-padrão $\left(S=2,9 \mathrm{~kg} / \mathrm{m}^{2}\right)$, a menor estimativa da diferença desejada entre a média amostral e a populacional (erro amostral; $\mathrm{d}=0,7$ $\mathrm{kg} / \mathrm{m}^{2}$ ) para garantir maior precisão, adotando-se $o$ nível de significância de $1 \%$, sendo $\mathrm{Z}$ o escore de uma distribuição normal padrão. Por conveniência e equilíbrio dos grupos, recomendou-se o maior n para cada um dos cinco estágios de desenvolvimento das mamas, que foi de 114 meninas obtido em Ml (estágio impúbere ou pré-adolescente para maturação de mamas).

Além do peso (obtido pela TANITA TBF-305) e da estatura (medida pelo antropômetro DEXTER 200), tomados de acordo com padrões internacionais, dos quais derivaram o IMC, as meninas foram questionadas a respeito da ocorrência da menarca pelo método status quo, que considera se até a data cada uma teve, ou não, a primeira menstruação (menarca: sim ou não). Para as que a referiram, foi solicitada a data do evento (método recordatório), para avaliar o tempo decorrido desde a menarca. O método status quo foi escolhido para o cálculo da idade mediana da menarca por estar menos sujeito a erros que o recordatório, uma vez que não depende da memória da informante. O estágio maturacional foi determinado por autoavaliação comparativa com pranchas ilustrativas do desenvolvimento das mamas (estágios de Tanner), sendo que, para validar essa avaliação, nas que consentiram $(84,51 \%)$, o dado foi reavaliado pela pesquisadora. Para analisar a concordância entre os observadores, foi utilizado o coeficiente de Kappa ponderado.

As escolas convidadas a participar foram escolhidas por sorteio e nos dois momentos obedeceu-se a mesma ordem para contato de adesão. Foram incluídas as alunas que tiveram o consentimento livre e esclarecido do diretor e dos responsáveis e que concordaram em participar, após terem sido consultadas antes da tomada das medidas.

O IMC foi analisado considerando-se os pontos de corte para magreza, eutrofia, sobrepeso e obesidade das Curvas da Organização Mundial da Saúde (OMS) 
2007. Para a análise da idade da menarca, o grupo sem excesso de peso (magreza + eutrofia) foi comparado com o grupo que apresentava excesso de peso (sobrepeso + obesidade).

Os dados foram processados no programa Statistical Analysis System (SAS), versão 9.2. Após a análise descritiva das variáveis, foram utilizados o teste do Qui-quadrado, para comparar proporções, e o de Mann-Whitney, para comparar as medidas numéricas entre os dois grupos. No cálculo da idade da menarca, o ajuste da curva sigmoide obtida da distribuição percentual da menarca "sim" foi feito pelo método logito [log (proporção de menarca presente/1- proporção de menarca presente)] obtendo-se uma reta. Com base na equação da reta, foi calculada a mediana aplicando-se a transformação logarítmica. Foram calculados os IC95\% para a idade da menarca e a probabilidade de $50 \%$ da ocorrência do evento. A regressão logística foi empregada para verificar se havia diferença significante da idade da menarca entre 2001 e 2010 (período considerado) e entre as meninas com e sem excesso de peso (diagnóstico nutricional). O mesmo método foi utilizado para analisar se havia interação entre esses dois efeitos (período e diagnóstico nutricional), ou seja, se a idade da menarca teria avançado por conta do aumento da prevalência de excesso de peso. O nível de significância adotado foi de $5 \%$.

Esta pesquisa seguiu os princípios da Declaração de Helsinque e teve a aprovação do Comitê de Ética da PUC-Campinas (protocolo 693/09).

\section{RESULTADOS}

A tabela 1 contempla a distribuição das 1.435 meninas avaliadas de acordo com o estágio maturacional de Tanner para mamas (M) e o número de meninas que referiram menarca por estágio maturacional, e a tabela 2 mostra a análise descritiva do Z-escore da estatura e IMC, idade e idade da menarca de acordo com o diagnóstico nutricional pelos pontos de corte para o IMC das curvas da OMS 2007. O teste de Kappa mostrou alta concordância $(0,96$; IC95\% 0,94-0,97) entre a autoavaliação e a avaliação feita pela pesquisadora, $o$ que permitiu a inclusão de todas as meninas medidas. Para as que se autoavaliaram, esse estágio foi considerado e, para as que se deixaram avaliar, foi considerado o estágio definido pela pesquisadora.

Não houve diferença significante na distribuição das meninas por estágio maturacional $(\mathrm{p}=0,2333)$ entre
2001 e 2010. Tanto a idade mediana das meninas nas duas amostras (12,5 anos em 2001 e 12,3 anos em 2010; $\mathrm{p}=0,0591)$ quanto o número de adolescentes que referiram menarca nos dois momentos -358 $(52,3 \%)$ em 2001 e $400(53,3 \%)$ em 2010; $\mathrm{p}=0,6849$ - foram semelhantes. A mediana do tempo decorrido entre a menarca e a coleta de dados foi de 2,14 anos em 2001 e 2,11 anos em 2010.

A diferença significativa $(\mathrm{p}=0,0041)$ em relação ao diagnóstico nutricional das meninas avaliadas em 2001 e 2010 deve-se principalmente à diminuição do número de eutróficas e ao aumento de obesas.

A tabela 3 contempla a idade mediana da menarca avaliada pelo logito. Nota-se uma antecipação de 3,24 meses na idade da menarca entre 2001 e 2010 ( $\mathrm{p}=$ $0,0143)$.

Quando a idade no evento foi comparada de acordo com o diagnóstico nutricional (Tabela 4), observa-se que nas duas amostras (2001 e 2010) as meninas com IMC maior (sobrepeso + obesidade) menstruaram mais cedo do que as sem excesso de peso. No período considerado, a menarca adiantou 1,44 mês no grupo das meninas magras e eutróficas e 5,76 meses nas com sobrepeso e obesidade.

Houve diferença da idade da menarca entre as meninas com e sem excesso de peso, tanto em 2001 $(\mathrm{p}=0,0214)$ quanto em $2010(\mathrm{p}<0,0001)$. Isoladamente, tanto o período avaliado - 2001 e $2010-(\mathrm{p}=$ $0,0102)$ quanto o diagnóstico nutricional $(\mathrm{p}=0,0201)$ mostraram influenciar a idade da menarca. No entanto, esses efeitos são independentes, pois sua interação (período*diagnóstico nutricional) não foi significante $(\mathrm{p}=0,1221)$.

Tabela 1. Distribuição das 1.435 meninas avaliadas em escolas particulares de Campinas-SP, de acordo com os estágios de maturação de Tanner para mamas e número de meninas que referiram menarca por estágio maturacional em 2001 e 2010

\begin{tabular}{|c|c|c|c|c|}
\hline \multirow{2}{*}{$\begin{array}{l}\text { Estágio de } \\
\text { Tanner para } \\
\text { mamas }^{\star}\end{array}$} & \multicolumn{2}{|c|}{2001} & \multicolumn{2}{|c|}{2010} \\
\hline & n $(\%)^{\dagger}$ & $\begin{array}{c}n \text { menarca } \\
(\%)^{\epsilon}\end{array}$ & $n(\%)^{\dagger}$ & $\begin{array}{c}n \text { menarca } \\
(\%)^{\epsilon}\end{array}$ \\
\hline M1 & $117(17,1)$ & 0 & $138(18,4)$ & 0 \\
\hline M2 & $150(21,9)$ & $6(4)$ & $135(18)$ & $2(1,5)$ \\
\hline M3 & $141(20,6)$ & $82(58,2)$ & $145(19,3)$ & $70(48,3)$ \\
\hline M4 & $150(21,9)$ & $143(95,3)$ & $193(25,7)$ & $189(97,9)$ \\
\hline M5 & $127(18,5)$ & $127(100)$ & $139(18,5)$ & $139(100)$ \\
\hline Total & 685 (100) & 358 & 750 (100) & 400 \\
\hline
\end{tabular}


Tabela 2. Análise descritiva das meninas avaliadas em 2001 e 2010 em escolas particulares de Campinas-SP, de acordo com 0 diagnóstico nutricional

\begin{tabular}{|c|c|c|c|}
\hline $\begin{array}{l}\text { Diagnóstico } \\
\text { nutricional }^{*}\end{array}$ & Variável & 2001 & 2010 \\
\hline \multirow[t]{6}{*}{ Magreza } & $\mathrm{n}$ do grupo $(\%)^{\dagger}$ & $9(1,3 \%)$ & $5(0,6 \%)$ \\
\hline & n com menarca $(\%)^{\epsilon}$ & $4(1,1 \%)$ & $2(0,5 \%)$ \\
\hline & Z-IMC & $-2,3 \pm 0,4$ & $-2,3 \pm 0,4$ \\
\hline & Z-estatura ${ }^{\varepsilon}$ & $-0,1 \pm 1,5$ & $-0,1 \pm 1$ \\
\hline & Idade ${ }^{£}$ (anos) & $13,6 \pm 2,7$ & $13,6 \pm 1,7$ \\
\hline & Idade da menarca ${ }^{£}$ (anos) & $12,8 \pm 1,2$ & $13,6 \pm 0,4$ \\
\hline \multirow[t]{6}{*}{ Eutrofia } & $\mathrm{n}$ do grupo (\%) ${ }^{\dagger}$ & $494(72,1 \%)$ & $499(66,5 \%)$ \\
\hline & n com menarca $(\%)^{€}$ & $274(76,5 \%)$ & $280(70 \%)$ \\
\hline & Z-IMC & $-0,2 \pm 0,7$ & $-0,2 \pm 0,7$ \\
\hline & Z-estatura ${ }^{\varepsilon}$ & $0,1 \pm 0,9$ & $0,1 \pm 1$ \\
\hline & Idade $^{£}$ (anos) & $13 \pm 2,2$ & $12,9 \pm 2,9$ \\
\hline & Idade da menarca (anos) & $11,9 \pm 1,1$ & $11,9 \pm 1,2$ \\
\hline \multirow[t]{6}{*}{ Sobrepeso } & $\mathrm{n}$ do grupo (\%) ${ }^{\dagger}$ & $145(21,2 \%)$ & $171(22,9 \%)$ \\
\hline & n com menarca $(\%)^{\epsilon}$ & $69(19,3 \%)$ & $90(22,5 \%)$ \\
\hline & Z-IMC & $1,4 \pm 0,3$ & $1,5 \pm 0,3$ \\
\hline & Z-estatura ${ }^{£}$ & $0,5 \pm 1,1$ & $0,4 \pm 0,9$ \\
\hline & Idade ${ }^{£}$ (anos) & $12,2 \pm 1,9$ & $12,1 \pm 2,5$ \\
\hline & Idade da menarca (anos) & $11,4 \pm 1,1$ & $11,6 \pm 1,2$ \\
\hline \multirow[t]{6}{*}{ Obesidade } & $\mathrm{n}$ do grupo (\%) ${ }^{\dagger}$ & $37(5,4 \%)$ & 75 (10\%) \\
\hline & n com menarca $(\%)^{\epsilon}$ & $11(3,1 \%)$ & $28(7 \%)$ \\
\hline & Z-IMC & $2,4 \pm 0,4$ & $2,4 \pm 0,3$ \\
\hline & Z-estatura ${ }^{£}$ & $0,6 \pm 1,2$ & $0,8 \pm 1$ \\
\hline & Idade ${ }^{£}$ (anos) & $11,9 \pm 2,1$ & $11 \pm 2,6$ \\
\hline & Idade da menarca (anos) & $12,2 \pm 0,9$ & $11 \pm 1,3$ \\
\hline \multirow[t]{2}{*}{ Total } & $\mathrm{n}$ meninas & 685 & 750 \\
\hline & $\mathrm{n}$ meninas com menarca & 358 & 400 \\
\hline
\end{tabular}

${ }^{*} p=0,0041$

t: \% do total; є: \% das meninas com menarca; \&: média \pm DP; DP: desvio-padrão.

Tabela 3. Idade mediana da menarca, calculada pelo logito (status quo), de alunas de escolas particulares de Campinas-SP avaliadas em $2001 \mathrm{e}$ 2010, com os IC95\% para a probabilidade e idade da menarca

\begin{tabular}{lccc}
\hline Ano (n) & $\begin{array}{c}\text { Idade mediana da } \\
\text { menarca } \\
\mathbf{P} \text { (menarca) = 50\% }\end{array}$ & $\begin{array}{c}\text { IC95\% para a } \\
\text { probabilidade }\end{array}$ & $\begin{array}{c}\text { IC95\% para a } \\
\text { idade da } \\
\text { menarca }\end{array}$ \\
\hline $2001(685)$ & 12,35 anos & $44 \%-56 \%$ & $12,21-12,48$ anos \\
$2010(750)$ & 12,08 anos & $43 \%-57 \%$ & $11,91-12,24$ anos \\
\hline
\end{tabular}

$p=0,0143$

Tabela 4. Idade da menarca, calculada pelo logito, de alunas de escolas particulares de Campinas-SP, avaliadas de acordo com o diagnóstico nutricional em 2001 e 2010

\begin{tabular}{lcccc}
\hline Ano & $\begin{array}{c}\text { Diagnóstico } \\
\text { nutricional }\end{array}$ & $\mathbf{n}$ & $\begin{array}{c}\text { Idade mediana } \\
\text { da menarca } \\
\text { (anos) }\end{array}$ & p-valor* \\
\hline 2001 & Magreza + eutróficas & 503 & 12,45 & 0,0214 \\
& Sobrepeso + obesidade & 182 & 12,08 & \\
2010 & Magreza + eutróficas & 504 & 12,33 & $<0,0001$ \\
& Sobrepeso + obesidade & 246 & 11,60 & \\
\hline
\end{tabular}

* Regressão logística.

\section{DISCUSSÃO}

A menarca adiantou 3,24 meses em 10 anos na população estudada, passando de 12,35 anos em 2001 para 12,08 anos em 2010. Houve aumento da obesidade, cujo percentual praticamente dobrou, e diminuição do número de eutróficas, enquanto a magreza e o sobrepeso pouco variaram. Nos dois períodos, a menarca ocorreu mais cedo no grupo de meninas com excesso de peso. A antecipação do evento foi maior nesse grupo ( 5,76 meses) se comparado às meninas sem excesso de peso (1,44 mês), mostrando que a obesidade está contribuindo para a antecipação da menarca. A ausência de interação entre o período e diagnóstico nutricional, no entanto, indica que a menarca antecipou não só pela mudança do perfil nutricional da amostra. Outros fatores, não considerados neste estudo, continuam contribuindo para esse avanço, o que sugere que o "potencial" dessa população pode ainda não ter sido atingido.

A esse respeito Ong e cols. comentam que não se deve imaginar que exista um "potencial mínimo", geneticamente determinado, para a idade da menarca para cada população, ou seja, uma idade mínima para a ocorrência do evento (11). Segundo esses autores, fatores ambientais, tais como localização geográfica, clima, alimentação e costumes, podem determinar uma ampla variação da idade da menarca em torno da média, como foi observado no Avon Longitudinal Study of Parents and Children (ALSPAC) desenvolvido no Reino Unido ( $13 \pm 1,7$ anos) (16). Além desses, o número de filhos na família, o salário, a ocupação e o nível educacional dos pais influenciam indiretamente a maturação e, consequentemente, a idade da menarca numa mesma população (17). Wonka também observou que a relação entre o IMC e a idade da menarca ocorre em todas as camadas sociais (18). O presente estudo abordou meninas de uma mesma camada social (alunas de escolas particulares) com o intuito de diminuir o viés. A antecipação foi maior no grupo com excesso de peso, corroborando os dados de outras pesquisas que apontam que a menarca ocorre mais cedo nas meninas obesas $(8,18)$.

Nas últimas décadas, as estatísticas sobre a saúde dos brasileiros melhoraram bastante, principalmente no que se refere à saúde infantil (19). São poucos, no entanto, os estudos nacionais realizados com amostras da mesma base populacional que permitem estabelecer a tendência temporal de diferentes indicadores de saúde.

Uma revisão da literatura identificou três pesquisas que abordam a tendência secular da menarca no Brasil. Kac e cols., analisando dados sobre a idade da menarca, colhidos no Rio de Janeiro (método recordatório), de 1.955 mulheres nascidas entre 1920 e 1979, constataram um 
avanço de 8,04 meses nesse período (aproximadamente 1,34 mês/década) (20). Os autores atribuíram essa tendência, que fez a idade da menarca diminuir de 13,07 para 12,40 anos em 60 anos, à melhora das condições de vida e saúde da população. Outro estudo, desenvolvido por Junqueira do Lago e cols. também no Rio de Janeiro, do qual participaram 2.217 mulheres nascidas entre 1932 e 1977, avaliou a idade da menarca obtida pelo método recordatório de acordo com o nível educacional dos pais, apontando 12,3 anos $\left( \pm \_1,64 \mathrm{DP}\right)$ como idade mediana para o evento e a antecipação de 2,4 meses/década (21). Essa tendência, no entanto, foi maior (3,6 meses) para o grupo com pais com escolaridade inferior a oito anos de estudo e menor (1,2 mês) para os com mais de oito, mostrando que, nas últimas décadas, a melhora nas condições de vida e saúde determinou um efeito mais forte na redução da idade da menarca nas mulheres de menor condição socioeconômica. Analisando a idade da menarca na população cabocla das margens do rio Amazonas e seus afluentes (164 mulheres de 8-19 anos e 158 de 20-92 anos), obtida pelo método recordatório nas mais velhas e status quo nas mais novas, Silva e Padez observaram uma antecipação de 19,44 meses entre as mulheres nascidas em 1930 (14,5 anos) e as nascidas em 1980 (12,88 anos), com média de 3,88 meses/década (22).

Nota-se, portanto, que o avanço da menarca varia conforme as variáveis preditoras do estudo. Analisando esses resultados, observa-se que os três estudos são transversais, englobam um período longo, tanto anterior como posterior à década de 1970, quando a melhora das condições nutricionais da população passou a influir de modo expressivo no avanço da menarca, e utilizaram o método recordatório que oferece viés de memória. Deve-se considerar ainda, ao se comparar os resultados, que a melhora das condições de vida e saúde no Brasil, observada principalmente após o início da década de 1970, não atingiu uniformemente todas as regiões do país, dificultando a comparação de resultados obtidos em estudos realizados na região Sudeste com o realizado na região Norte. Nesse sentido, apesar de analisar uma amostra de conveniência, a presente pesquisa procurou minimizar alguns vieses seguindo o mesmo protocolo nos dois momentos, utilizando o método status quo para obter a informação sobre a ocorrência da menarca (que é mais confiável do que o recordatório) e incluindo apenas adolescentes.

Historicamente, a antecipação da puberdade sempre foi relacionada à melhora das condições nutricionais (23). Mais recentemente se tem questionado se a antecipação da puberdade é causa ou consequência do aumento da massa corporal (2), pois estudos mostram que as meni- nas nos Estados Unidos estão entrando em puberdade mais cedo, se comparadas há 30 anos, ao mesmo tempo que a prevalência da obesidade triplicou no país $(13,24)$. Dunger e cols. comentam, no entanto, a complexidade dessa associação, pois talvez não seja só a obesidade um fator de risco para a antecipação da puberdade, mas outros fatores, entre os quais citam os constituintes da dieta (9). A associação entre excesso de adiposidade e maturação fez com que se levantasse hipótese sobre aspectos endócrinos relacionados ao tecido adiposo (24), pois esse tecido, contribuindo para a aromatização dos andrógenos em estrógeno, adiantaria a maturação.

Dois estudos longitudinais, com desenhos distintos, que examinaram o papel da obesidade na tendência secular da maturação sexual chegaram a conclusões diferentes. Demerath e cols. avaliaram a tendência do IMC em relação à idade da menarca e a curva de crescimento das meninas que participaram do Fels Longitudinal Study $(25,26)$. Esse estudo abrange 50 anos. A idade da menarca nas meninas nascidas na década de 1970 (12,58 anos) foi 5 meses mais baixa do que nas nascidas nas 4 décadas anteriores $(\mathrm{p}>0,05)$ e 3 meses maior $(12,34$ anos $)$ do que nas meninas nascidas na década de $1980(\mathrm{p}<0,05)$. No mesmo período, no entanto, não houve aumento correspondente significante do IMC aos 3, 6 e 9 anos e na menarca. Assim, a análise mostrou que a antecipação da menarca não se associou a um aumento do IMC nas coortes avaliadas de acordo com o ano de nascimento. Para esses autores, o aumento do peso é consequência e não o determinante da idade da menarca; eles sugerem que as mudanças seculares do IMC e da idade da menarca são fenômenos independentes, embora coincidentes. Já Anderson e cols. pesquisaram a relação entre IMC, raça e idade da menarca com base nos dados do NHES (National Health Examination Survey) (27). Estudando a probabilidade estimada da menarca de acordo com a idade, a mediana da menarca no NHANES III ( The Third National Health and Nutrition Examination Survey) foi predita a partir dos dados do IMC de fato observado no NHANES III. Os autores, então, compararam a idade predita (12,56 anos) com a idade da menarca observada no estudo NHANES III (12,54 anos) e concluíram que houve uma diferença de apenas 7 dias indicando que o avanço da menarca entre os dois estudos está de acordo com a mudança observada na distribuição e valores do IMC. Para Anderson e cols., a queda de 2,52 meses na média da idade da menarca entre os períodos 1963 1970 (NHES ciclos II e III; 12,75 anos) e 1988-1994 (NHANES III; 12,54 anos) foi paralela ao aumento do $\mathrm{Z}$-escore do IMC acima do percentil 85 , que passou de 
$16 \%$ para $27 \%$ (27). Esse aumento, quando ajustado para a idade e raça, associou-se com a probabilidade aumentada da menarca. Nota-se então que, enquanto Demerath e cols., usando os dados do Fels, concluíram que o avanço da menarca não está relacionado à tendência secular do IMC $(25,26)$, Anderson e cols., analisando os dados do NHES e NHANES III, constataram haver forte evidência que liga a mudança do IMC à idade da menarca (27).

Embora o presente estudo compare duas coortes transversais, os dados mostraram que os efeitos - período e diagnóstico nutricional - são independentes, ou seja, não foi só o aumento da prevalência de excesso de peso que determinou o avanço da idade da menarca. As meninas com excesso de peso menstruaram mais cedo nos dois períodos avaliados e a antecipação também foi maior nesse grupo, mas outros fatores não considerados nesse estudo estão contribuindo para a antecipação do evento, pois entre as eutróficas a menarca também está ocorrendo mais cedo. É importante, no entanto, notar que a ocorrência da menarca foi avaliada retrospectivamente, de tal forma que não se conhece o IMC por ocasião do evento e sim na data da coleta de dados. Assim, embora o tempo decorrido entre a menarca e a tomada das medidas não tenha sido grande nos dois períodos ( 2 anos), deve-se ter cautela ao interpretar uma relação de causa-efeito.

A obesidade praticamente dobrou (passando de 5,4\% para $10 \%$ ), acompanhando a tendência que vem ocorrendo em países desenvolvidos (6). Esse quadro preocupa não só pelo risco de doenças, mas também por expor mais cedo as meninas com sobrepeso e obesidade, que maturam mais cedo, à vida sexual e, consequentemente, ao risco de gravidez. Medidas devem ser tomadas tanto no que diz respeito ao controle do ganho de peso como no sentido de orientá-las quanto a doenças sexualmente transmissíveis e métodos contraceptivos. Novas pesquisas devem abordar outros aspectos que possam estar influenciando na antecipação da menarca, tanto nas meninas eutróficas quanto nas com excesso de peso.

Agradecimentos: à reitoria da PUC-Campinas pelas bolsas de Iniciação Científica (FAPIC/Reitoria).

Declaração: os autores declaram não haver conflitos de interesse científico neste estudo.

\section{REFERÊNCIAS}

1. Euling SY, Herman-Giddens ME, Lee PA, Selevan SG, Juul A, Sorensen TI, et al. Examination of US puberty-timing data from 1940 to 1994 for secular trends: panel findings. Pediatrics. 2008;121(Suppl 3):S172-91.

2. Kaplowitz PB. Link between body fat and the timing of puberty. Pediatrics. 2008;121(Suppl 3):S208-17.
3. Morris DH, Jones ME, Schoemaker MJ, Ashworth A, Swerdlow AJ. Secular trends in age at menarche in women in UK born 190893: results from the Breakthrough Generations Study. Paediatr Perinat Epidemiol. 2011;25(4):394-400.

4. Biro FM, Khoury P, Morrison JA. Influence of obesity on timing of puberty. Int J Androl. 2006;29(1):272-7.

5. Nour NN. Obesity in resource-poor nations. Rev Obstet Gynecol. 2010;3(4):180-4.

6. Prentice AM. The emerging epidemic of obesity in developing countries. Int J Epidemiol. 2006;35(1):93-9.

7. Solorzano $\mathrm{CMB}, \mathrm{McC}$ artney $\mathrm{CR}$. Obesity and the puberal transition in girls and boys. Reproduction. 2010;140(3):399-410.

8. Wang Y. Is obesity associated with early maturation? A comparison of the association in American boys versus girls. Pediatrics. 2002;110(5):903-10.

9. Dunger DB, Ahmed ML, Ong KK. Effects of obesity on growth and puberty. Best Pract Res Clin Endocrinol Metab. 2005;19(3):375-90.

10. Kaplowitz PB. Pubertal development in girls: secular trends. Curr Opin Obstet Gynecol. 2006;18(5):487-91.

11. Ong KK, Ahmed ML, Dunger DB. Lessons from large population studies on timing and tempo of puberty (secular trends and relation to body size):The European trend. Mol Cell Endocrinol. 2006;254-255:8-12.

12. Biro FM, Wien M. Childhood obesity and adult morbidities. Am J Nutr. 2010;91(5):1499S-505S.

13. Lee JM, Appugliese D, Kaciroti N, Corwyn RF, Bradley RH, Lumeng JC. Weight status in young girls and the onset of puberty. Pediatrics. 2007;119(3):e624-30.

14. Colli AS. Crescimento e desenvolvimento pubertário em crianças e adolescentes brasileiros, VI: maturação sexual. São Paulo: Editora Brasileira de Ciências; 1988.

15. Anjos LA, Veiga GA, Castro IRR. Distribuição dos valores do índice de massa corporal da população brasileira até 25 anos. Pan Am J Public Health. 1998;3(3):164-73.

16. Golding J, Pembrey M, Jones R, ALSPAC Study Team. ALSPAC - the Avon Longitudinal Study of Parents and Children. I. Study methodology. Paediatr Perinat Epidemiol. 2001;15(1):74-87.

17. Castilho SD, Barros-Filho AA. Crescimento pós-menarca. Arq Bras Endocrinol Metab. 2000;44(3):195-204.

18. Wonka I. Association between BMI and age at menarche in girls from different socio-economic groups. J Biol Clinc Anthop. 2010;68(1):43-52.

19. Monteiro CA. Children's health: trends and determinants in S. Paulo city, Brazil, in the second half of $20^{\text {th }}$ century. Rev Saude Publica. 2000;34(6 Suppl):1-4.

20. Kac G, Coel ASC, Velasquez-Melendez G. Secular trend in age at menarche for women born between 1920 and 1979 in Rio de Janeiro, Brazil. Ann Hum Biol. 2000;27(4):423-8.

21. Junqueira do Lago M, Faerstein E, de Souza Lopes C, Werneck GL. Family socio-economic background modified secular trends in age at menarche: evidence from the Pro-Saude Study. Ann Hum Biol. 2003;30(3):347-52.

22. Silva HP, Padez C. Secular trend in age at menarche among $\mathrm{Ca}-$ boclo populations from Pará, Amazonia, Brazil: 1930-1980. Am J Hum Biol. 2006;18(1):83-92.

23. Davison KK, Susman EJ, Birch LL. Percentage body fat at age 5 predicts earlier pubertal development among girls at age 9 . Pediatrics. 2003;111(4 pt 1):815-21.

24. Himes $\mathrm{JH}$. Examining the evidence for recent secular changes in the timing of puberty in US children in light of increases in the prevalence of obesity. Mol Cell Endocrinol. 2006;254-255:13-21.

25. Demerath EW, Li J, Sun SS, Chumlea WC, Remsberg KE, Czerwinski SA, et al. Fifty-year trends in a serial body mass index during adolescence in girls: the Fels Longitudinal Study. Am J Clin Nutr. 2004;80(2):441-6.

26. Demerath EW, Towne B, Chumlea WC, Sun SS, Czerwinski SA, Remsberg KE, et al. Recent decline in age at menarche: the Fels Longitudinal Study. Am J Hum Biol. 2004;16(4):453-7.

27. Anderson SE, Dallal GE, Must $A$. Relative weight and race influence average age at menarche: results from two nationally representative surveys of US girls studied 25 years apart. Pediatrics. 2003;111(4 Pt 1):844-50. 